\title{
Tunable Diplexer for Cognitive Radio RF Front-end Modules
}

\author{
Erick Emmanuel Djoumessi ${ }^{1, *}, \mathrm{Ke} \mathrm{Wu}^{2}$ \\ ${ }^{1}$ Intel Corporation, Libertyville, 60048, Illinois, United States \\ ${ }^{2}$ Poly-Grames Research Center, Ecole Polytechnique de Montreal, Montreal, H3T1J4, Quebec, Canada \\ *Corresponding Author: erickemmanuel.djoumessi@polymtl.ca
}

Copyright (C) 2014 Horizon Research Publishing. All rights reserved.

\begin{abstract}
This paper describes the design and measurement of a varactor-tuned planar diplexer for reconfigurable multi-mode/multi-standard transceiver front-end modules. The proposed diplexer of $3 \mathrm{~cm} \times 1.8 \mathrm{~cm}$ is composed of two tunable dual-mode bandpass filters with two varactor diodes. The diplexer channels are $8.8 \%$ relative bandwidth at 2.48 and $3.2 \mathrm{GHz}$ for $25 \mathrm{~V}$ biased voltage, while $7.5 \%$ and $4.1 \%$ are obtained at 2.075 and $2.606 \mathrm{GHz}$ for $5 \mathrm{~V}$, respectively. The center-frequency tunability for lower and upper channel filters bands are $21.4 \%$ and $20.4 \%$, respectively. Measured insertion loss varies from 2.4 to $4.8 \mathrm{~dB}$ and 2.8 to $5 \mathrm{~dB}$ for the two channels over the tuning frequency range with better than 30-dB isolation. The lower channel filter (with higher capacitance) presents a better 1-dB compression measurement point $(\mathrm{P}-1 \mathrm{~dB})$ over the tuning frequency range.
\end{abstract}

Keywords Tunable Diplexer, Dual-Mode Filter, Tunable Transceiver, Varactor Diode, Cognitive Radio, SDR

\section{Introduction}

Bluetooth, IEEE802.16 and IEEE802.11a/b/g wireless radio technologies for personal area networks (PAN), wireless metropolitan area networks (WMAN) and wireless local area networks (WLAN), have been developed to meet different cellular phone service requirement. Thus, wireless platforms are equipped with more and more radio transceivers operating in parallel on chips to satisfy those needs. However, the power consumption, cost and interference between radios chipsets are major problems encountered by those systems. An emerging solution is related to the development of reconfigurable microwave components to realize radio systems that can dynamically detect available channel access in order to improve overall spectrum usage. In [1], a Ka-band ferroelectric thin film/dielectric diplexer was realized as a critical part of a discriminator-locked tunable oscillator. Moreover, tunable RF front-end components that have largely been studied and implemented for the future software defined cognitive radio systems (SDCR) are RF filters [2], [3], couplers [4], [5] ], and demodulators [6]. In this letter, a varactor-tuned planar dual-mode bandpass filter is used to design a tunable diplexer. Simulations and measurements results of S-parameters and 1-dB compression point for different biased voltage are also presented.

\section{Varactor-Tuned Dual-Mode Bandpass Filter}

Fig. 1(a) shows the structure of the proposed tunable diplexer. It consists of two capacitively loaded second-order microstrip dual-mode bandpass filters (BPF) and a tee junction connecting the individual BPFs to a common input port 1. Let the upper and lower filters be named filter 1 and filter 2, respectively. The microstrip dual-mode resonator is widely used to design bandpass filters due to its compact size and selectivity. A tunable dual-mode bandpass filter was presented in [3]. However, very little effort has been focused on its detailed analysis. For our analysis purpose, such a square microstrip dual-mode resonator can be represented as a resonant cavity with $\mathrm{TM}_{\mathrm{mn} 0}^{\mathrm{z}}$ modes. The analytical expressions of EM fields within the cavity have well been detailed in [7]. According to the boundary conditions, the analytical expressions of electric and magnetic fields within the cavity are given by Eq. (1), where indexes $m, n$ characterize modes and $\mathrm{kc}, \omega$ are respectively the propagation constant and the angular frequency. Amn and eeff stand for mode magnitude and dielectric effective permittivity.

$$
\begin{gathered}
E_{z}=\sum_{m=0}^{\infty} \sum_{n=0}^{\infty} A_{m n} \cos \left(\frac{m \pi}{a} x\right) \cos \left(\frac{m \pi}{a} y\right) \\
H_{x}=\left(\frac{j \omega \varepsilon_{\text {eff }}}{k_{c}^{2}}\right)\left(\frac{\partial E_{z}}{\partial y}\right) \\
H_{y}=-\left(\frac{j \omega \varepsilon_{\text {eff }}}{k_{c}^{2}}\right)\left(\frac{\partial E_{z}}{\partial x}\right)
\end{gathered}
$$




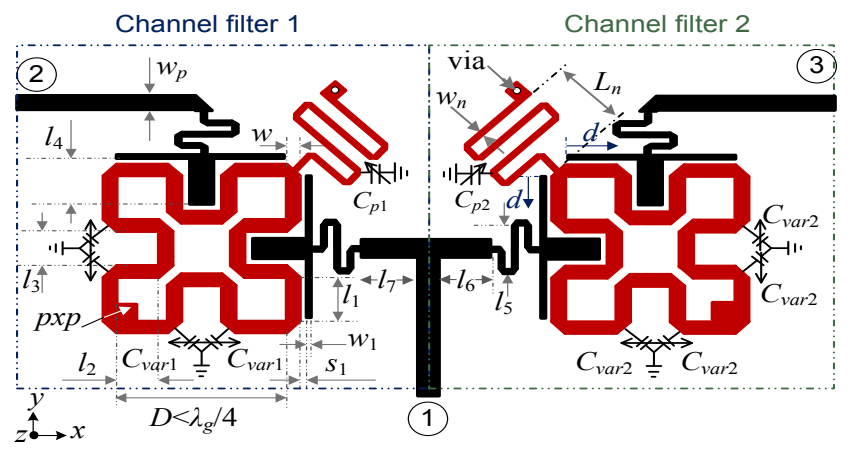

(a)

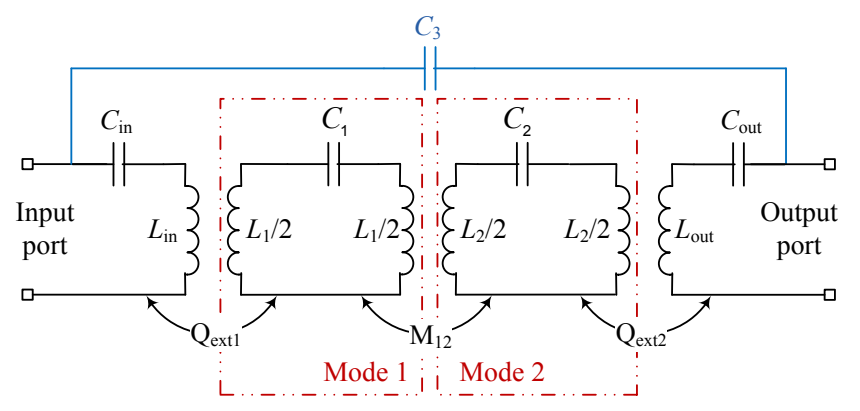

(b)

Figure 1. (a) Layout of the proposed tunable diplexer based-on two identical two-pole dual-mode bandpass filter loaded with two different capacitances, (b) equivalent circuit of a dual-mode bandpass filter

The first resonant degenerated modes TM100 and TM010 within the cavity are characterized by the frequency given as $\mathrm{fc}=2 / \lambda \mathrm{g}$ ( $\mu \varepsilon$ eff) $1 / 2$, where $\lambda \mathrm{g}$ is the guided wavelength evaluated at the center operation frequency, and ceff stands for dielectric effective permittivity. To illustrate and facilitate the design principle of the tunable diplexer, Fig. 1(b) presents a circuit model of the two pole dual-mode BPF (as shown in Fig. 1(a)). Both degenerated modes are represented by $\mathrm{L} 1,2 \mathrm{C} 1,2$, series resonators, magnetically coupled through coupling coefficient M12, used to model the perturbation pxp in combination with capacitor $\mathrm{Cp} 1,2$. Cross-coupling capacitor $\mathrm{C} 3$ models the direct coupling between the input and output (I/O) ports (as shown in Fig 1(a) by variable d) and it is used to create a pair of transmission zeros for improving the dual-mode filter selectivity. The external quality factor Qext1= Qext2 [8] can be found from coupling gap s1 and line width w1.

To validate the proposed circuit model, the tunable filter 1 is designed at $\mathrm{fl}=3.2 \mathrm{GHz}$ by using EM software ADS Momentum [9]. The substrate used is Rogers RT/Duroid 6010 with thickness of $0.635 \mathrm{~mm}$ and relative permittivity $\varepsilon \mathrm{r}=10.2$. Geometrical dimensions are $\mathrm{wp}=0.593 \mathrm{~mm}, \mathrm{w} 1=$ $0.1523 \mathrm{~mm}, \mathrm{w}=0.3 \mathrm{~mm}, 11=2.43 \mathrm{~mm}, 12=1.3 \mathrm{~mm}, 13=1.2$ $\mathrm{mm}, 14=1.7 \mathrm{~mm}, 15=3.5 \mathrm{~mm}, \mathrm{~s} 1=0.1524 \mathrm{~mm}, \mathrm{wn}=0.15$ $\mathrm{mm}, \mathrm{ln}=3.2 \mathrm{~mm}$ and Cvar $1=\mathrm{Cp} 1=0.45 \mathrm{pF}$. Circuit model [see Fig. 1(b)] parameters values are given as: $\mathrm{Lin}=\mathrm{Lout}=$ $12.4 \mathrm{nH}, \mathrm{Cin}=$ Cout $=0.22 \mathrm{pF}, \mathrm{L} 1=\mathrm{L} 2=10 \mathrm{nH}, \mathrm{C} 1=\mathrm{C} 2=0.25$ $\mathrm{pF}, \mathrm{M} 12=0.04, \mathrm{C} 3=0.1 \mathrm{pF}$. Analytical expressions of the external/unloaded quality factors (Qext, Qu) [4], and coupling coefficients $\mathrm{K} 12$ are given by:

$$
\begin{gathered}
k_{12}=\frac{f_{02}^{2}-f_{01}^{2}}{f_{02}^{2}+f_{01}^{2}} \\
Q_{e x t}=\frac{f_{0}}{f_{2}-f_{1}} \\
Q_{u}=\frac{Q_{e x t}}{\left(1-10^{-I L / 20}\right)}
\end{gathered}
$$

where $\mathrm{f} 01$ and $\mathrm{f} 02$ represent the two resonant frequencies modes TM100 and TM010. f0, f2-f1 and IL (insertion loss) are for the center operation frequency, 3-dB bandwidth and insertion loss in $\mathrm{dB}$, respectively. Fig. 2 shows the computed values of $\mathrm{Q}$ and $\mathrm{K} 12$ against gap s1 and for different values of Cvar 1 and $\mathrm{Cp} 1$. As the loaded capacitances Cvar1= $\mathrm{Cp} 1=$ Cvar increase with a constant value of s1, the external Qext increases (i.e: 3-dB bandwidth decreases and Qu increases) (see Fig. 2(a)). However, the insertion loss increases with the loaded Cvar, as a consequence of mismatch. Maximum values of $\mathrm{Qu}=96,110,117$ are obtained at different $\mathrm{s} 1=0.05$, $0.16,0.26 \mathrm{~mm}$ for various $\mathrm{Cvar}=0.45,0.65$ and $0.85 \mathrm{pF}$, respectively. Fig. 2(a) shows that Q-factor at different Cvar, can be controlled by simply tuning gap $\mathrm{s} 1$. On the other hand, ratio $\mathrm{f} 02 / \mathrm{f} 01$ and coefficient $\mathrm{K} 12$ against capacitance $\mathrm{Cp} 1$ are shown in Fig. 2(b) for different values of Cvar $1=0.45$ and $0.65 \mathrm{pF}$, respectively. To keep a constant $\mathrm{K} 12=\mathrm{M} 12=$ 0.04 (3-dB bandwidth) at different center-operation frequency defined by Cvar $=0.45$ and $0.85 \mathrm{pF}$, the value of Cp1 should be 0.425 to $0.92 \mathrm{pF}$ (see Fig. 2(b)).

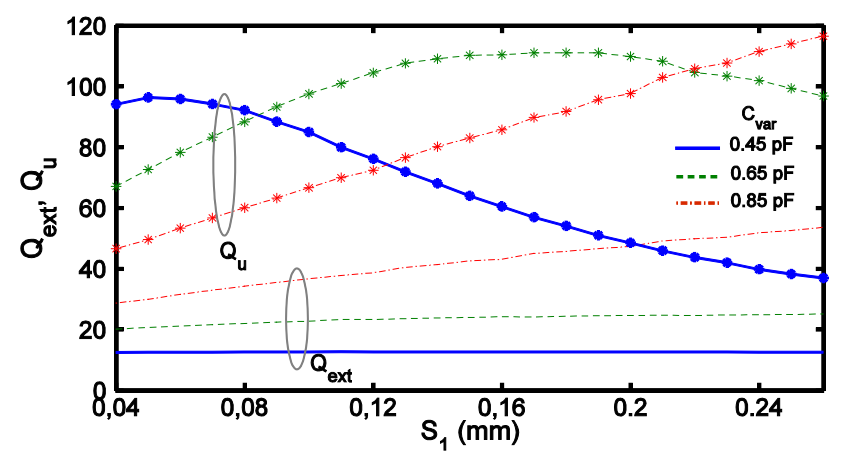

(a)

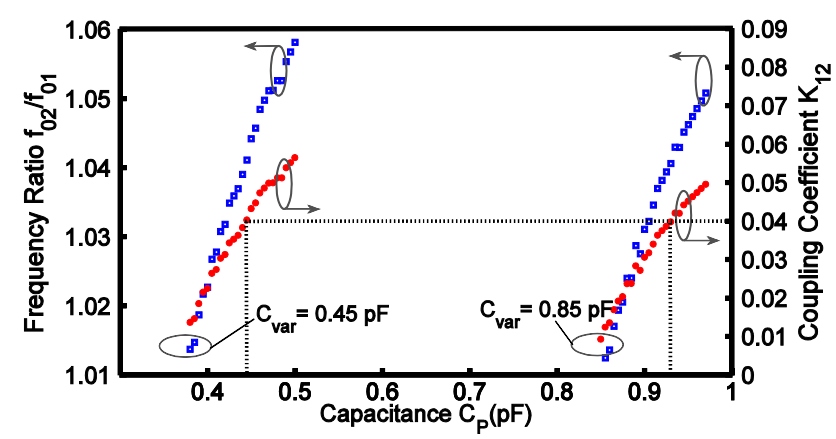

(b)

Figure 2. (a) External and unloaded quality factor, (b) two degenerate mode frequencies and their coupling coefficients for different values of loading capacitances $C_{\mathrm{var}}$ and $C_{\mathrm{p}}$. 
Typical simulated responses of the circuit and (EM) model as shown in Fig. 3(a). Both models show responses with a pair of transmission zeros at the edge of the filter bandpass for various values of $\mathrm{C} 3(0.01,0.05,0.1 \mathrm{pF})$ and $\mathrm{d}(0,1,2$ $\mathrm{mm}$ ) to demonstrate the influence of the transmission zeros on the insertion loss. When the value of $\mathrm{C} 3$ increases (d decreases), the upper and lower stopband transmission zeros will be closer to the passband edges with the stable passband insertion loss that is essentially not degraded. The circuit model shows a broadband frequency response than the EM model. This is mainly due to the ideal lossless lumped elements used in the circuit model that result to an $I L=0$. Eq. (2) also shows that as IL increase, the $3-\mathrm{dB}$ bandwidth decrease (see Fig. 3(a)).
Finally, capacitances Cvar1 and $\mathrm{Cp} 1$ are replaced by identical varactor diodes, denoted by $\mathrm{C}$. The chip varactor diode used is the MSV34,069 from Aeroflex Metelics, with capacitance variation from 0.4 to $2.5 \mathrm{pF}$ over a $30-\mathrm{V}$ bias voltage with equivalent resistance of $0.8 \Omega$. As shown in Fig. 3(b), simulated and measured results (S21 and S11) at different bias voltage are in good agreement. The measured insertion losses at the center operation frequency vary from 2.2 to $5.2 \mathrm{~dB}$ with $20.7 \%((\mathrm{fmax}-\mathrm{fmin}) /(\mathrm{fmax}+\mathrm{fmin}) / 2)$ center-frequency tunable range from approximately 2.6 to 3.2 GHz. The frequency shift observed between the simulation and measurement results are mainly due to the wire bonding effect used to connect the varactor diode to the microstrip line.

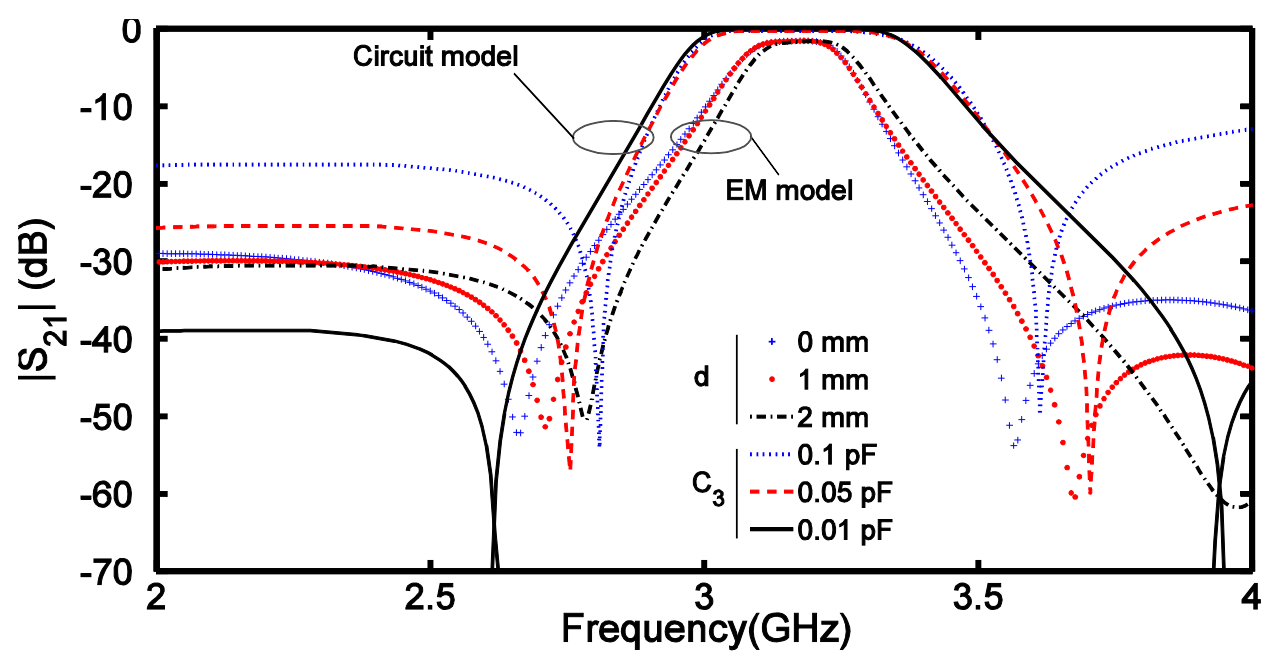

(a)

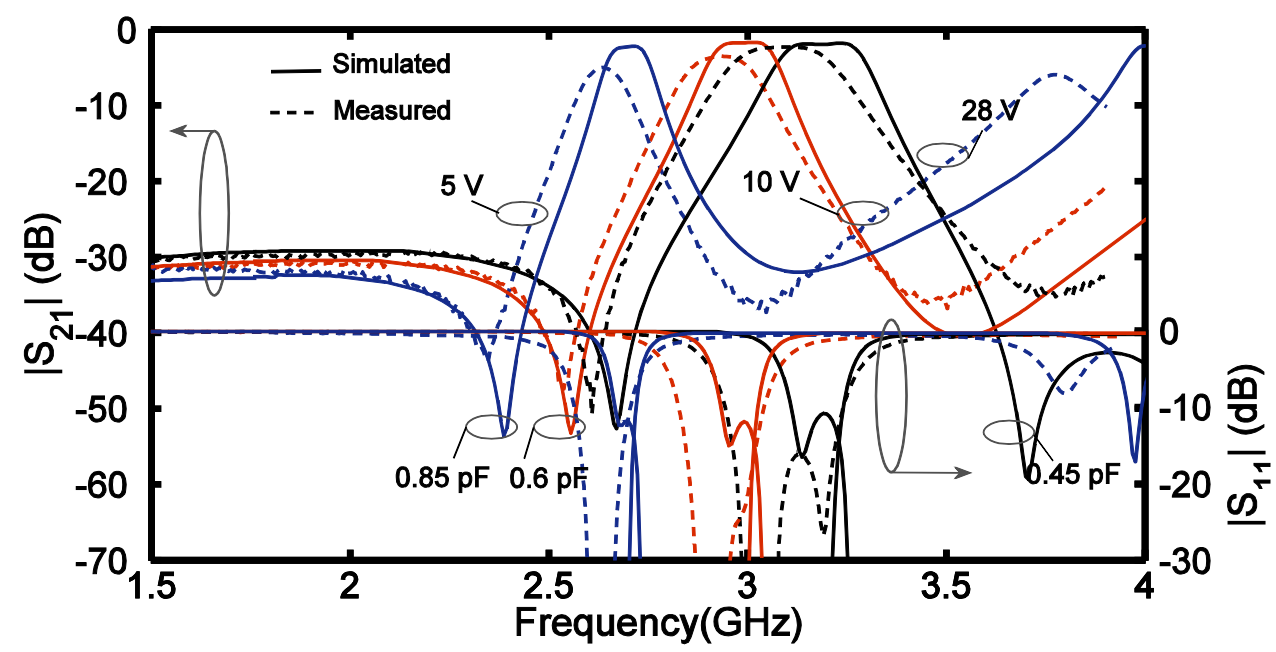

(b)

Figure 3. (a) Circuit model and EM simulated frequency responses of a dual-mode BPF for various values of $\mathrm{C}_{3}$ and $d$ with $M_{12}=0.04$ and $C_{\mathrm{p}}=C_{\mathrm{var}}=0.45 \mathrm{pF}$, (b) EM simulated and measured frequency responses of the tunable dual-mode bandpass filter for various $V_{\mathrm{b}}, C_{\mathrm{var}}=C_{\mathrm{P}}=C$ 


\section{Electronically Tunable Diplexer}

A tunable dual-mode BPF has been designed and experimentally validated for the diplexer upper channel band by using a varactor diode MSV34069. The same procedure is used to design the lower channel filter 2 with the center frequency at $\mathrm{f} 2=2.6 \mathrm{GHz}$. A varactor diode MSV34075, with capacitance variation from 0.7 to $4 \mathrm{pF}$ over a 30 -V bias voltage with equivalent resistance of $0.5 \Omega$, is used to reduce the filter size and thus the same bias voltage supply $(\mathrm{Vb})$ as for the upper channel filter 1 is also preserved. Geometrical dimensions of tunable BPF 2 are the same as for the filter 1, except length $14=2.4 \mathrm{~mm}$ and capacitance $\mathrm{Cvar} 2=\mathrm{Cp} 2=0.8$ $\mathrm{pF}$. Connection of both tunable BPF 1 and 2 to the common input port 1 is realized by T-junction with physical lengths $16=6 \mathrm{~mm}$ and $17=7.7 \mathrm{~mm}$. Fig. 4 shows the fabricated tunable diplexer prototype. Simulation and measurement S-parameters are shown in Figs. 5 for different capacitances Cvar1,2 and biased voltage $\mathrm{Vb}$. Bias voltage range $\mathrm{Vb}$, corresponding to the tuning capacitance range (from 0.45 to $0.85 \mathrm{pF}$ for MSV34069 and from 0.8 to $1.2 \mathrm{pF}$ for MSV34075), covers about 25 to 5 volt. The tunable upper and lower channel filters exhibit $20.4 \%$ and $21.4 \%$ tunable range from approximately 2.606 to $3.2 \mathrm{GHz}$, and 2.075 to $2.48 \mathrm{GHz}$. The measured $3-\mathrm{dB}$ bandwidth in the tuning range is around $8.8 \%$ at 2.48 and $3.2 \mathrm{GHz}$ for $25 \mathrm{~V}$ biased voltage, while $7.5 \%$ and $4.1 \%$ are obtained at 2.075 and $2.606 \mathrm{GHz}$ for $5 \mathrm{~V}$, respectively. The measured insertion loss losses (S31 and S21) vary from 2.4 to $4.8 \mathrm{~dB}$ and 2.8 to $5 \mathrm{~dB}$ for the two channels over the tuning frequency range with better than 30-dB isolation (S23). Such insertion losses are mainly due to the combination of substrate dielectric loss and silicon varactor diode loss that increases as the bias voltage decreases (capacitance increases). The measured return loss (S11, S22, and S33) at the common port 1, output port 2 and 3 are from -7 to $-20 \mathrm{~dB}$ in the upper and lower passbands.

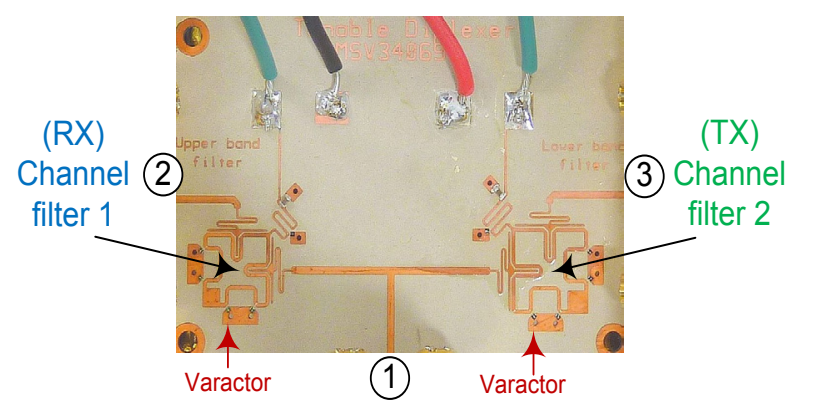

Figure 4. Photograph of fabricated tunable diplexer

Since varactor diodes MSV34069 and MSV34075 present nonlinear characteristics, a RF input signal tone (Pin) level is applied at common port 1 of the diplexer and the output signal (Pout) level is measured at ports 2 and 3. Fig. 6 shows the measured Pout versus $P$ in for bias voltage $\mathrm{Vb}=25$ and 10 volt, corresponding to the lower/upper center-operation frequency of $2.2 / 2.8 \mathrm{GHz}$ and $2.51 / 3 \mathrm{GHz}$, respectively. As shown in Fig. 6, the Pout signal level saturation suggests a better 1-dB compression point (as well as linearity) and insertion loss for the lower channel BPF 2 (loaded with diode MSV34075), than its upper counterpart with varactor MSV34069. These results suggest the usefulness of BPF 2 and BPF 1 as transmit and receive channel pasbands, respectively.

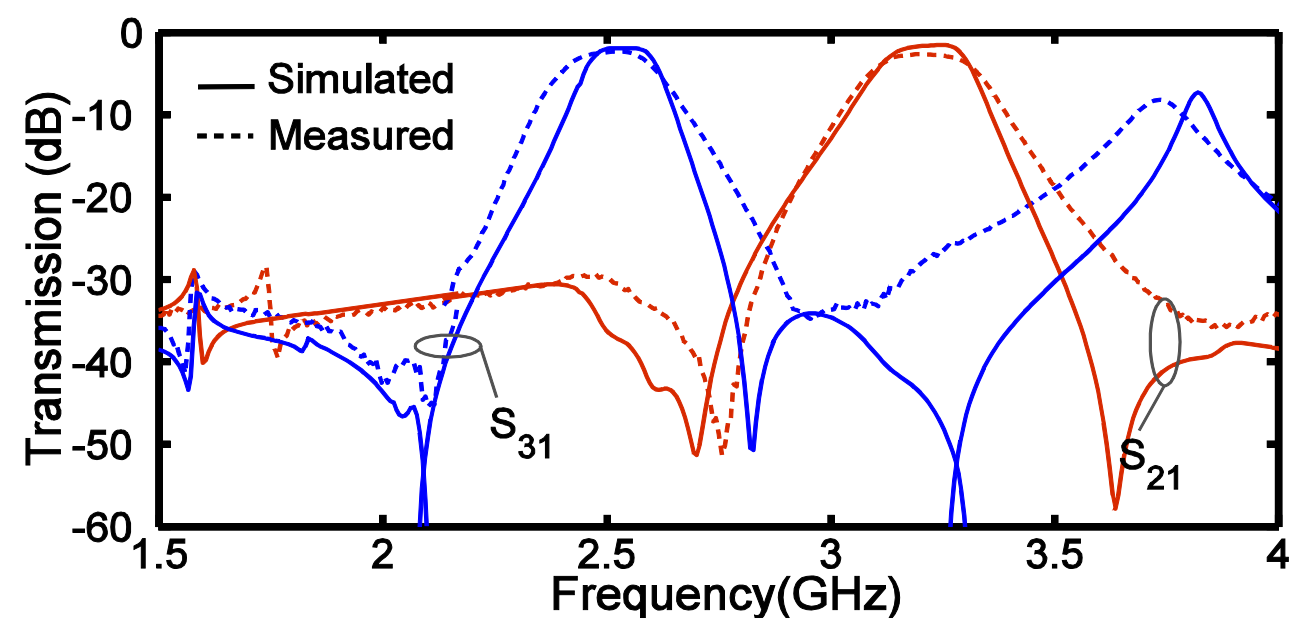

(a) 


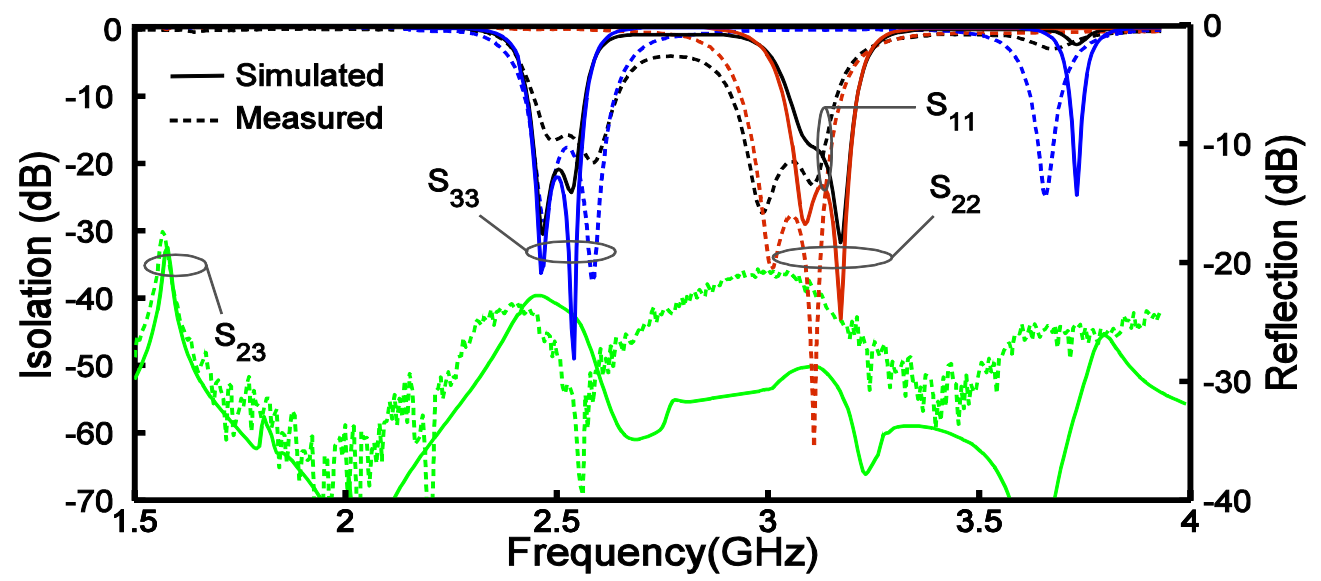

(b)

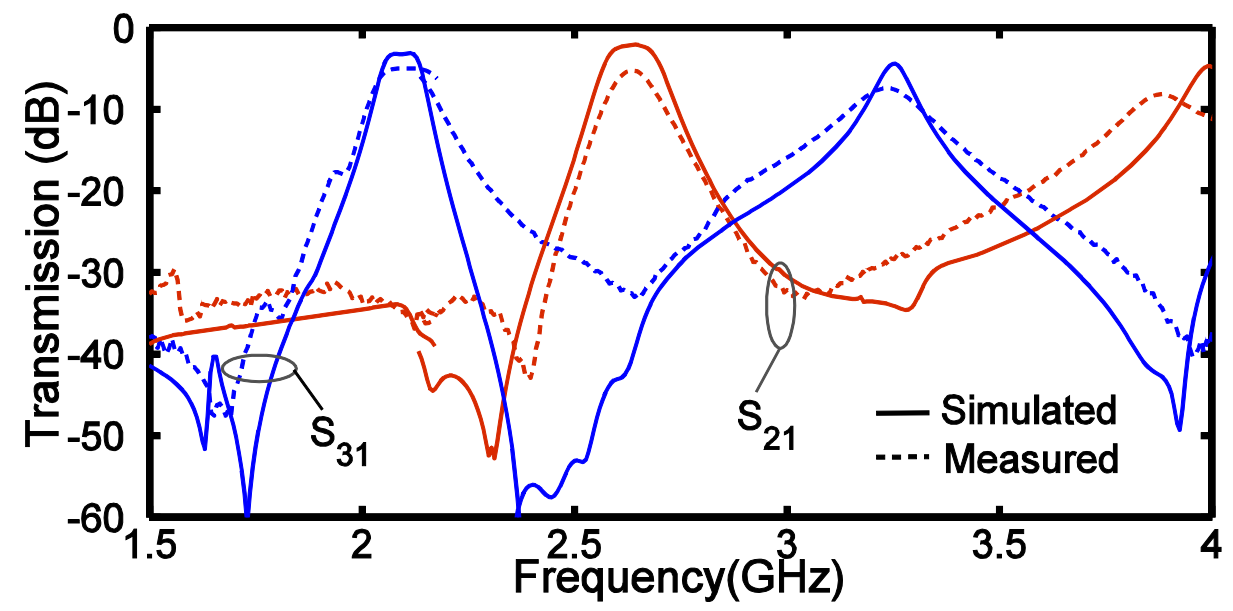

(c)

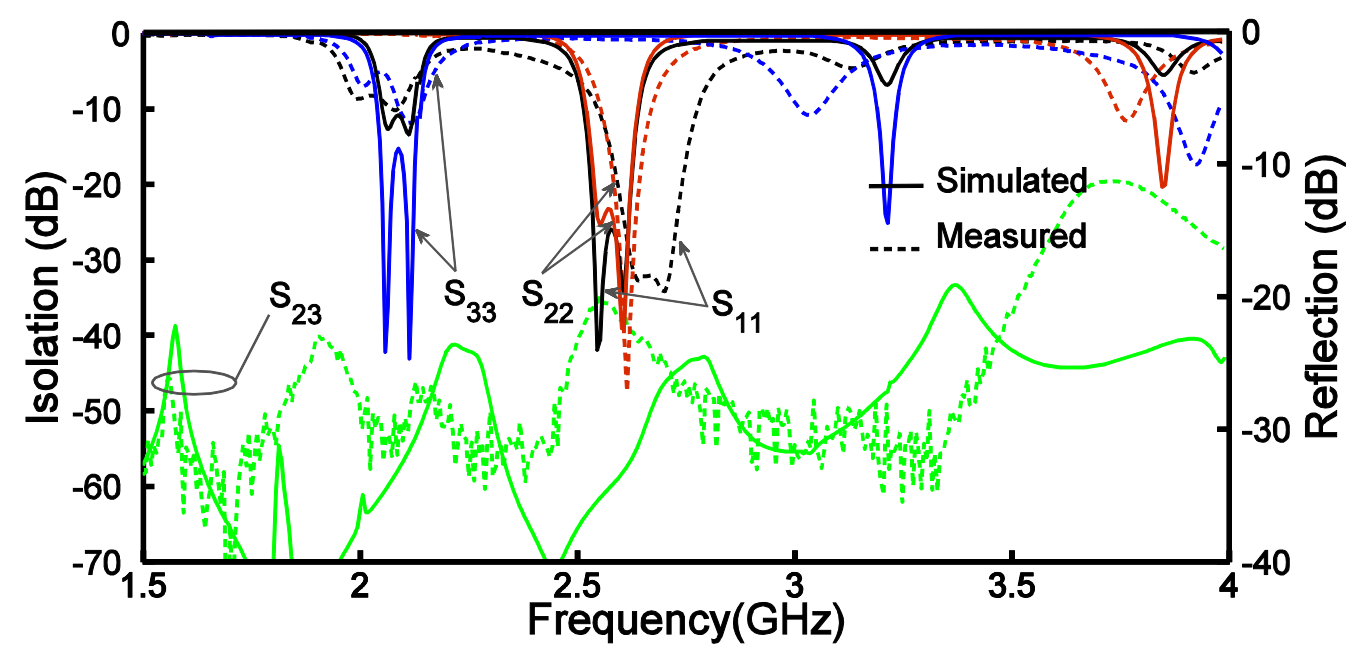

(d)

Figure 5. Simulated and measured results of magnitudes of $S_{11}, S_{21}, S_{31}, S_{22}, S_{33}$ and $S_{23}$ for ((a), (b)) $\mathrm{C}_{\mathrm{var} 1}=\mathrm{C}_{\mathrm{p} 1}=0.45 \mathrm{pF}, \mathrm{C}_{\mathrm{var} 2}=\mathrm{C}_{\mathrm{p} 2}=0.8 \mathrm{pF}, V_{\mathrm{b}}=25 \mathrm{v}$, and ((c), (d)) $\mathrm{C}_{\mathrm{var} 1}=\mathrm{C}_{\mathrm{p} 1}=0.85 \mathrm{pF}, \mathrm{C}_{\mathrm{var} 2}=\mathrm{C}_{\mathrm{p} 2}=1.2 \mathrm{pF}, V_{\mathrm{b}}=5 \mathrm{v}$. 


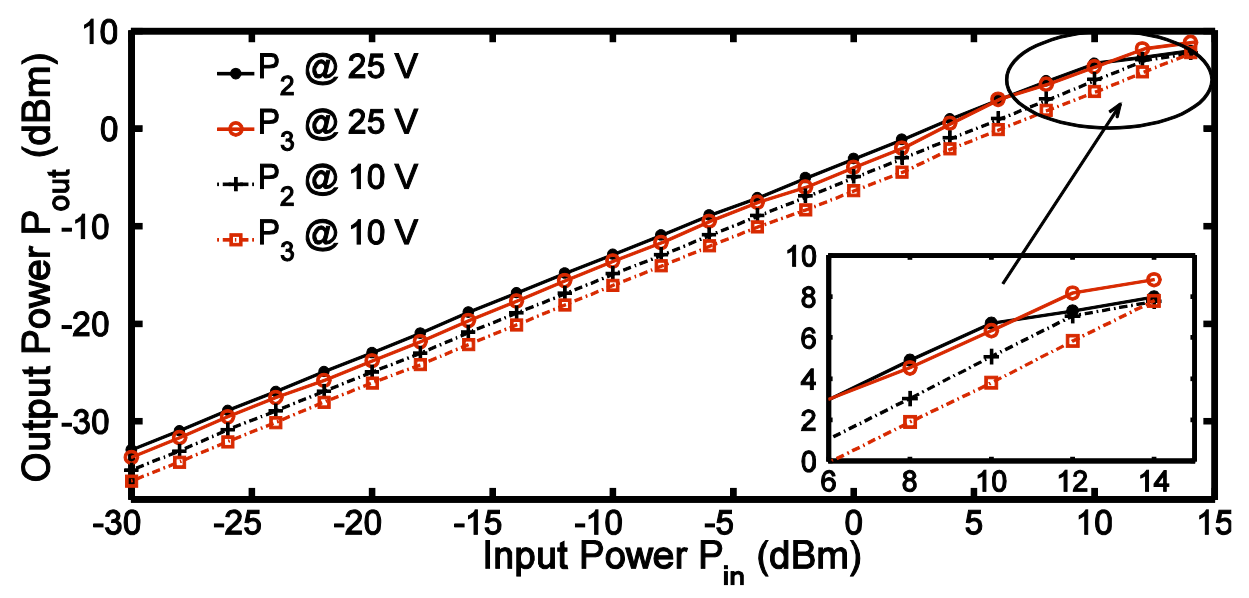

Figure 6. Output power level $P_{\text {out }}$ at ports 2 and 3 against input power level $P_{\text {in }}$ at port 1, for different biased voltage $V_{\mathrm{b}}=10,25 \mathrm{v}$.

\section{Conclusion}

A frequency-agile diplexer is proposed and experimentally validated by using tunable dual-mode bandpass filters. The diplexer compact size of $3 \mathrm{~cm} \times 1.8 \mathrm{~cm}$ is achieved with two types of silicon varactor diode. Both adjacent channel center-frequency tunabilities are $21.4 \%$ and $20.4 \%$ with isolation better than $30 \mathrm{~dB}$. The proposed diplexer can be used for tunable multi-mode/multi-standard transceiver systems.

\section{Acknowledgements}

The authors thank the technical personal of Poly-Grames Research Center, Ecole Polytechnique de Montreal, in the fabrication and mounting of the diplexer prototype.

\section{REFERENCES}

[1] F., A. Miranda, G. Subramanvam, K. Van, F. W. Romanofsky, "A K-band (HTS gold)/ferroelectric thin film/dielectric diplexer for a discriminator locked tunable oscillator," IEEE Trans. Applied Supercon., vol. 9, no. 2, pp. 3581-3584, June 1999.

[2] M. A. El-Tanani, and G. M. Rebeiz, "A two-pole two-zero tunable filter with improved linearity," IEEE Trans. Microw. Theory Tech., vol. 57, no. 4, pp. 830-840, April. 2009.

[3] E. E. Djoumessi, M. Chaker, and K. Wu, "Varactor-tuned dual-mode bandpass filter for wireless applications," in Radio Wireless Conf., Jan. 2009, pp. 646-649.

[4] M., H., Lehmann, T., Knoechel, "Systematic design of reconfigurable quadrature directional couplers," IEEE MTT-S, pp. 1009-1012, June. 2009.

[5] E. E. Djoumessi, and K. Wu, "Varactor-tuned multiband six-port front-end for wireless applications," Asia-Pacific Conf., pp. 1-5, Dec. 2008.

[6] E. E. Djoumessi, and K. Wu, "Tunable multi-band direct conversion receiver for cognitive radio systems," IEEE MTT-S, pp. 217-220, Jun. 2009.

[7] J.S Hong, M. J. Lancaster, Microstrip Filters for RF/Microwave Applications, New York: Wiley, 2001.

[8] K. Chang, L. H. Hsieh, Microwave Ring Circuits and Related Structures: New York: Wiley, 2004.

[9] 'Advanced design system', Palo Alto, CA, 2006. 